\title{
Design and Creation of Custom Internet of Things (Iot) Device for Analysis of Drunk and Drowsy Driver Detection
}

\author{
Prakhar Jain, Yeshveer Singh Yadav, Nilay Khare
}

\begin{abstract}
Road Safety is the most crucial aspect in this modern world as the reckless use of the developed technology i.e. vehicles, is leading to human loss. Safety concerns while driving is of utmost importance in our daily lives. It might lead to the loss of lives of even innocent people and loss of property. The evolution of the Internet of Things (IoT) and the continuing increase in the number of sensors connected to the Internet has led to development of various application in our day to day life. Thus, automation in road safety using IoT can help to reduction as well as prevention of accidents. Accuracy is important when it comes to road safety and one must always strive to achieve the highest accuracy, provided there is no trade off, with inexcusable time. Most of

the road accidents are caused due to drowsiness or drunk driving. This paper deals with the design and testing of drunk and sleep-deprived driver detection device. This paper presents a low-cost effective method for drowsiness detection and proposes a method to detect whether the driver is drunk or not. Finally, it lays off a way to communicate with emergency help services. It deals with the design and testing of drunk and drowsy driver detection device.
\end{abstract}

Keywords: Drunk Detection, Internet of Things (IoT), Road Safety, Sleep Detection, Simple Mail Transfer Protocol (SMTP).

\section{INTRODUCTION}

According to the World Health Organization (WHO), approximately 1.35 million people die each year due to road accidents [1]. So, the issue of safety while driving is paramount in our daily lives. It might lead to loss of lives and loss of property. Around $90 \%$ of accidents are caused due to human errors. The problem will persist and is also on an exponential rise. Hence, it requires more solutions.

Distracted driving is the cause of the highest number of accidents. Some other causes are drinking, distractions like texting while driving and rubbernecking.

Revised Manuscript Received on April 18, 2020.

* Corresponding Author

Prakhar Jain*, Department of Computer Science and Engineering, Maulana Azad National Institute of Technology, Bhopal, India, prakharjain927@gmail.com.

Yeshveer Singh, Department of Computer Science and Engineering, Maulana Azad National Institute of Technology, Bhopal, India, yeshveersinghyadav@gmail.com.

Nilay Khare, Department of Computer Science and Engineering, Maulana Azad National Institute of Technology, Bhopal, India, nilay.khare@rediffmail.com.

(C) The Authors. Published by Blue Eyes Intelligence Engineering and Sciences Publication (BEIESP). This is an open access article under the CC BY-NC-ND license (http://creativecommons.org/licenses/by-nc-nd/4.0/)
Though drunk driving is illegal in most areas of the world but is accounted for one of the highest accounted reason for accidents. A lot of times, drunk driving victims aren't the ones who have been drinking. This may be the reason for the loss of even innocent lives. While most of the road accidents are also caused because of drowsiness (sleep deprivation) and working environments i.e. reduced sleep and time factor. Traffic survey shows that driver fatigue may be a contributory factor in up to $20 \%$ cases and due to alcohol drinking, it is about $31 \%$ cases of all road accidents. Sleep deprived driving is not something that happens rarely, in this competitive environment, stress is all over the mind, it happens every day of the year. So, the driver may fall asleep while driving the vehicle and may become the victim of the accident. Driver drowsiness and distracted driving reduce the driver's decision-making capability and perception level. These problems cannot be taken lightly as it concerns the loss of lives and property. Hence these factors are a reason for losing control over the vehicle and making the vehicle prone to accidents.

A deficiency of an efficient emergency contact service is also a reason for the loss of lives that can be saved in time. This is needed when a crash has occurred in a stranded region and there is a communication problem, then it could lead to loss of lives which could have been saved by an earlier response by emergency response services. Hence there needs to be an efficient, fast and reliable communication to emergency contact services after an accident.

Different methods have been evolving to make the road safer. Different technologies have made their ways to make road less prone to accident and to prevent the loss of lives and even property. But in recent times the use of IoT (Internet of Things) for road safety is at zenith. Internet of Things (IoT) is an ecosystem of connected physical objects that are accessible through the internet. The evolution of the Internet of things (IoT) and the continuing increase in the number of sensors connected to the Internet impose big challenges regarding the management of the resulting deluge of data and network latency. Uploading sensor data over the web does not add value. Therefore, an efficient knowledge extraction technique is badly needed to reduce the amount of data transfer and to help simplify the process of knowledge management [3]. In [4], the authors describe virtually endless opportunities and connections possible for IoT with proposing about a Smart City concept with Urban IoT architecture.

Published By:

Blue Eyes Intelligence Engineering

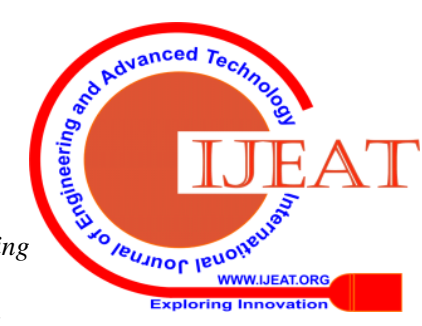


In [5], a synchronized concept of road map and IoT has been made wherein the task is to reduce off road collisions. In [6], the authors have presented a remotecontrolled car with limited automation features like traffic light detection, obstacle avoidance and lane detection system.

This paper presents a novel method which is a low-cost hardware IoT device which can be easily installed and be used in for area with less traffic density with the aim to reduce road trauma in relation to minor accidents. It does explain the solution in three steps i.e. Prevention (drunk detection), Avoidance (sleep detection) and Recovery (solution to contact emergency services at times of emergency). We aid to intend in road safety of people by bringing in a device that could detect whether the person is drunk or sleepy and thus could help in prevention of accidents. It could even send notifications at the occurrence of accident and inform about the accident, thus acting like emergency contact services. Thus, this device overcomes the limitation of previous approaches as discussed above.

\section{METHODOLOGY}

The flowchart, as in Fig. 1, shows the proposed methodology.

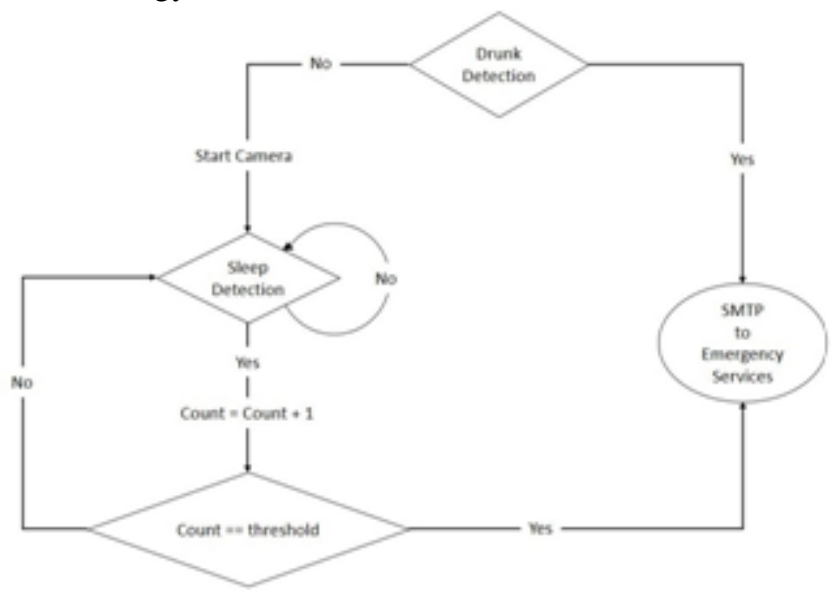

Fig. 1. Flowchart of Proposed Methodology

It describes the proposed algorithm as a solution for preventing accidents due to drunk driving (Prevention) or sleep deprived driving (Avoidance), while also providing a solution to the authorities and emergency services in case of emergency using SMTP protocol (Recovery).

\section{A. Drunk Detection}

A hardware solution has been proposed for detection of alcohol consumption. By implementing this a safe journey is possible by decreasing the accident rate due to drinking. Alcohol sensor MQ3 is suitable for detecting alcohol concentration with high sensitivity and fast response time.

Alcohol sensor (MQ3) works as a Breathalyzer and calculate blood alcohol content (BAC) from breath alcohol content (BrAC) and then using it predicts whether the driver is drunk or not. Arduino continuously checks alcohol content present in the air and computes blood alcohol content in percentage from it. If the calculated BAC crosses the threshold limit, at that time it will alarm through buzzer and will turn off the relay. If the alcohol consumption limit is over drunk that time, it will send mail/message through SMTP to emergency contact services [8]. Algorithm 1 below describes the hardware algorithm design for detection of alcohol consumption using MQ3 sensor.

Algorithm 1: MQ3 Drunk detection

Data: Driver's Breath

Result: Alcohol Level of Driver

1 Initialize Baud Rate;

2 MQ3 Pin = Input Pin;

3 Alarm Pin = Output Pin;

4 read analog value;

5 if analog value > LIMIT then

6 output = Alarm Pin;

7 go to SMTP;

8 else

$9 \quad$ go to line 4;

The LIMIT in algorithm 1 is set using the Table I.

Table- I: Different LIMIT and their outputs

\begin{tabular}{|c|c|c|c|}
\hline LIMIT & Level & Buzzer & SMTP \\
\hline $0-20 \%$ & Intoxicated & OFF & OFF \\
\hline $21-40 \%$ & Slightly Drunk & OFF & ON \\
\hline $41-60 \%$ & Drunkenness & ON & ON \\
\hline $61-99 \%$ & Over Limit Drunk & ON & ON \\
\hline
\end{tabular}

B. Detection of Sleep Driving

An algorithm has been designed to detect the drowsiness levels of a driver using eye detection. An eye blink is a fast closing and reopening of a human eye. Every person has a different eye blinking pattern. Quantifiable characteristics of eye blinking includes the length of a blink duration (usually between 100 and 400 milliseconds), the height and width of the eye opening, and the speed of which people close and open their eyes.

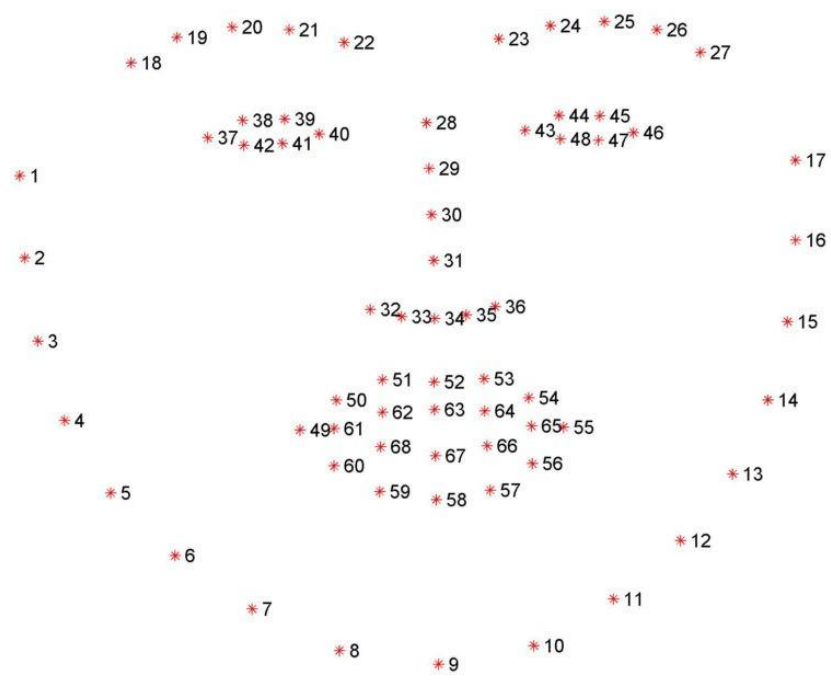

Fig. 2. Facial Landmarks

Published By:

Blue Eyes Intelligence Engineering \& Sciences Publication 
The algorithm 2 below is based on the concept on recognizing landmarks on the face. It has been done by using the 68 points facial landmarks done in dlib library shown in Fig. 2.

We propose to exploit state-of-the-art facial landmark detectors to localize the eyes and eyelid contours. From the landmarks detected in the image, we derive the eye aspect ratio (EAR) that is used as an estimate of the eye-opening state. Since the per frame EAR may not necessarily recognize the eye blinks correctly, a classifier that takes a larger temporal window of a frame into account is trained. The points corresponding to eyes i.e. 6 points for each eye in Fig. 2 are taken in the form of 3 and are used to calculate the threshold value of EAR which comes out to be 0.25 . The graph for different values of EAR [7] which is the average of EAR of left eye and right eye is shown in Fig. 3 which is calculated using (1).

$E A R=(\|p 2-p 6\|+\|p 3-p 5\|) /(2 *\|p 1-p 4\|)$

where $\mathrm{p} 1, \ldots, \mathrm{p} 6$ are the 2D landmark locations as

shown in the Fig. 3 below.

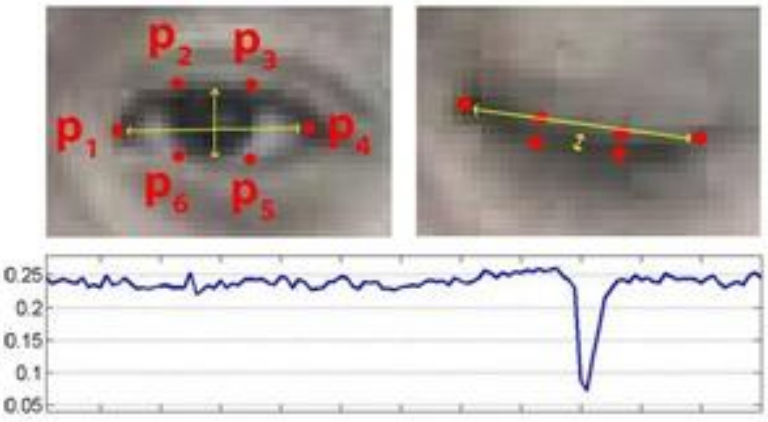

Fig. 3. Eye Aspect Ratio

The EAR is mostly constant when an eye is open and is getting close to zero while closing an eye. It is partially person and head pose insensitive. Aspect ratio of the open eye has a small variance among individuals, and it is fully invariant to a uniform scaling of the image and in-plane rotation of the face. Since eye blinking is performed by both eyes synchronously, the EAR of both eyes is averaged.

Algorithm 2: Drowsiness Detection

Data: Live Camera pictures by Camera Module

Result: Alarm when the driver is sleepy

1 import “dlib”, “cv2”, "numpy”, “scipy”,

"pygame" library;

2 Function start sound ()

3 play alarm sound;

4 Function eye aspect ratio $(X)$

$5 \quad \mathrm{~A}=$ Euclidean dist (p2, p6);

$6 \quad \mathrm{~B}=$ Euclidean dist (p3, p5);

$7 \quad \mathrm{C}=$ Euclidean dist (p1, p4);

8 ear $=(A+B) /(2 * C)$;

9 Start Camera;

10 Initialize Predictor Path;

//facial landmarks face classification 68 points

11 left = Convex Hull (LeftEye);

12 right = Convex Hull (RightEye);

13 ear left = eye aspect ratio(left);

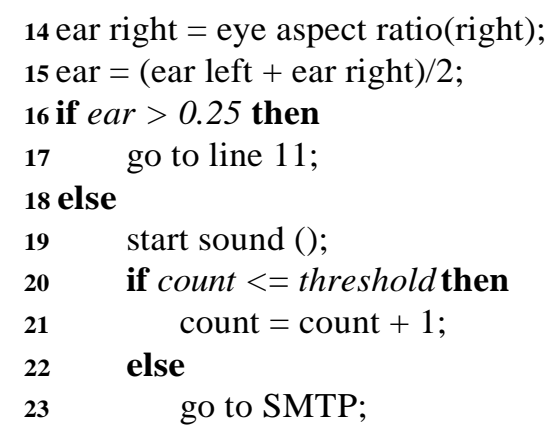

24 Close Camera;

Though the algorithm is primarily based on EAR [7], it is designed to be much more robust under a variety of conditions. To keep track of the eye, the algorithm assumes that the subject would remain stationary (except for their eyes) as the algorithm runs. In the proposed approach, a Viola Jones has been used as a face detector to detect the person's face and use heuristics to acquire the boxes as mentioned above. Thus, the modified approach detects the person's visage even if the person is moving. It works well even in case of drivers wearing spectacles and under low light conditions also.

It can detect the eye regions for blink detection. Additionally, it scales up or scales down the circumscribing box around the eye region according to if the person is moving away or near, respectively. If the person moves closer or further from the camera, the eye bounding box will adjust itself. Thus, making the proposed algorithm robust to scales.

\section{SMTP to Emergency Contact Services}

A SMTP system solves the problem of reporting the accident to the nearest emergency service provider like ambulance, fire brigade etc. This is needed when a crash has occurred in a stranded region and there is communication problem or a communication delay or gap, then it could lead to loss of lives which could have been saved by an earlier intervention by emergency response services. Hence, we need a faster and reliable emergency contact service for such cases. We have utilized SMTP (Simple Mail Transfer Protocol) for this purpose.

SMTP is an application layer protocol. The client who wants to send the mail opens a TCP connection to the SMTP server and then sends the mail across the connection. The SMTP server is always on listening mode. As soon as it listens for a TCP connection from any client, the SMTP process initiates a connection on that port 25 . After successfully establishing the TCP connection the client process sends the mail instantly [9]. Algorithm 3 below describes the working of modified SMTP protocol.

Algorithm 3: SMTP emergency contact mail services

Data: Content for mail

Result: Mail Sent Successfully

1 import "smtplib"
library;

Published By:

Blue Eyes Intelligence Engineering

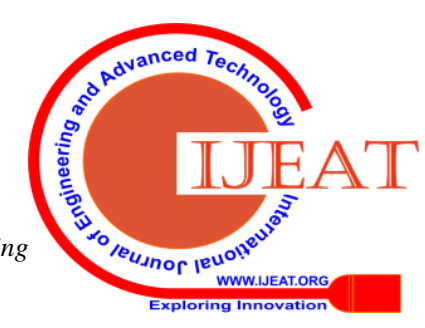


2 Enter login credentials (sender email, sender password);

3 if count $==$ threshold then

$4 \quad$ content $=$ Send for drowsiness or rubbernecking;

5 else

$6 \quad$ content $=$ Send for drunk driving;

7 end

$\mathbf{8}$ Enter receiver credentials (sender email, receiver email, content);

9 Close mail services;

Though the algorithm is based on SMTP [10], it is designed to be more robust according to variety of factors. It has been in designed in such a way that it sends mail according to the problem. In case for sleep deprivation or distraction it waits for a count (in Algorithm 1) to cross a certain threshold (set as 3 ). It also sends a mail in case if the MQ3 sensors shows the alcohol content of the driver crosses the LIMIT. Thus, it does inform the emergency contact services in case of drowsiness or drunk driving. Thus, being an important factor to prevent or lessen the accidents.

\section{RESULT AND DISCUSSION}

\section{A. Platform used for experiment}

A Raspberry Pi 3 Model B with Raspbian OS installed with SD card has been used. It also has CSI camera port for connecting a Raspberry Pi camera which has been used for sleep detection, DSI display port for connecting a Raspberry Pi touchscreen display which can be used to display messages to make the driver aware, 1 GB RAM for high speed computations which helps in detecting face faster as in the case of sleep detection algorithm [11]. For drowsiness detection a 5 MP Pi 3 Model B Camera has been used and for detection of alcohol consumption MQ3 sensor has been used. MQ3 sensor is highly sensitive to alcohol. It has fast response time and is stable and has long life [12]. Hence is one of the best hardware solutions for alcohol detection. A buzzer has also been used to alarm the driver either of sleep deprivation or drunk driving.

\section{B. Experiment performed}

The above proposed methodology has been categorized into 3 steps i.e. drunk detection (Prevention), detection of deprived sleep driving (Avoidance) and SMTP to emergency contact services (Recovery).

Drunk Driving has been proposed to be prevented by MQ3 sensor. The person who is drunk can be detected using MQ3 as it detects the alcohol consumption. Whenever the threshold limit set according to table I crosses, the buzzer beeps out. Different cases/scenarios for alcohol consumption detection were tried. Some of the scenarios are:

1. The driver was not drunk but had a concentrated breath. If the alarm does not buzz, then it shows that the MQ3 sensor is highly sensitive to alcohol.

2. People other than the driver were drunk for which getting no alarm would show that the MQ3 sensor senses alcohol in a certain range limited to the driver.

Different scenarios for sleep detection were tried on the person subjected to testing. The cases are as follows: 1.The person driving is continuously blinking his eyes. 2.The person driving is moving such that the camera needs precision to detect his face.

3. There is more than one face in the image taken by the camera.

4. The person is being distracted due to the surrounding.

For the case of SMTP protocol, it has been found to work successfully every time when there is a case of drunk detection or drowsiness detection. There is an automatic mail generation to the emergency contact services according to the flag raised. 


\section{Results}

As mentioned before, the drunk detection was tested on 100 persons with 37 of them had consumed alcohol and 63 individuals did not. To evaluate the performance of the model, this paper uses confusion matrix (also called an error matrix) which is a specific table layout that is used for the visualization of the performance of the supervised machine learning algorithms [13]. The outcomes obtained after hyperparameter tuning are shown in Table II.

Table- II: Confusion Matrix

\begin{tabular}{|c|c|c|}
\hline & Drunk & Not Drunk \\
\hline Drunk & $35(\mathrm{TP})$ & $2(\mathrm{FN})$ \\
\hline Not Drunk & $14(\mathrm{FP})$ & $49(\mathrm{TN})$ \\
\hline
\end{tabular}

So, the accuracy achieved by the proposed model is $84.00 \%$, precision is $71.42 \%$, recall is $94.59 \%$, F-score is $85.37 \%$ and G-mean is $85.78 \%$.

As mentioned in section III-B, the different scenarios of drowsiness detection were performed on a driver. After this experiment, it was found to be able to detect sleep for every case mentioned there. Similarly, for the above cases whenever there was a drunk or sleep detection there was an automatic mail generation by SMTP. Hence this IoT device works well with the cases mentioned about them.

\section{CONCLUSION}

There are many methods to monitor the driver and thereby alert him/her in case of distraction. But this paper provides a method/device to further increase road safety. It provides a way to prevent accidents. This method is a low-cost hardware IoT device which can be easily installed and used in areas with less traffic density to prevent minor accidents. It can be easily installed in vehicles to increase the safety without harming the vehicle and without being a distraction to the driver. To reduce road accidents there is also a need to alert the driver, which this device does precisely. Thus, this preliminary design is an efficient method to reduce road accidents. It has room for improvements.

In addition, future studies will focus on monitoring and detecting the driver's behavior. It is important to capture driver's behavior which will control the accidents due to rash driving. Further studies would also focus on improving the capability and reach of the algorithm to prevent road accidents and provide swiftness in emergency services communication. It will also be important to maintain a secure communication for reliable sending of distress or emergency messages.

\section{REFERENCES}

1. "Global Status Report On Road Safety 2018”, World Health Organization (WHO), 2018

2. T. Sivakumar and R. Krishnaraj, "Road Traffic Accidents (RTAs) due to Drunken Driving in India - Challenges in Prevention," IRACST- International Journal of Research in Management Technology (IJRMT), ISSN: 2249-9563 Vol. 2, No. 4, August 2012.

3. K. Ashton, “The Internet of Things Thing," RFID Journal, June 22, 2009

4. A. Zanella, N. Bui, A. Castellani, L. Vangelista and M. Zorzi, "Internet of Things for Smart Cities," IEEE Internet of Things Journal, Vol. 1, Issue 1, Feb. 2014

5. D. Vishal, H. S. Afaque, H. Bhardawaj and T. K. Ramesh, "IoTdriven road safety system," 2017 Inter- national Conference on Electrical, Electronics, Communication, Computer, and
Optimization Techniques (ICEECCOT), 15-16 Dec. 2017.

6. Md. R. T. Hossai, Md. A. Shahjalal and N. F. Nuri, "Design of an IoT based autonomous vehicle with the aid of computer vision,' 2017 International Conference on Electrical, Computer and Communication Engineering (ECCE), doi 10.1109/ECACE.2017.7913003, 16-18 Feb. 2017.

7. T. Soukupova and J. Cech, "Real-Time Eye Blink Detection using Facial Landmarks," 21st Computer Vision Winter Workshop, Slovenia, February 3-5, 2016.

8. P. H. Kulkarni, R. Wafgaonkar, S. S. Gujarathi and G. Ahirrao, "Alcohol Detection and Automatic Drunken Drive Avoiding System," International Journal of Engineering Research and Applications, Vol. 4, Issue 4, pp. 21-24, April 2014.

9. J. B. Postel, "Simple Mail Transfer Protocol," Information of Sciences Institute, University of Southern California, 1982.

10. J. Klensin, "Simple Mail Transfer Protocol," Network Working Group, October 2008

11. M. Richardson and S. Wallace, "Getting Started with Raspberry Pi," Maker Media Inc., CA, 2012.

12. “Technical Data MQ3 Gas Sensor,” Hanwei Electronics Co. Ltd.

13. M. Sokolova, and G. Lapalme, "A systematic analysis of performance measures for classification tasks," Information Processing Management, 45, (4), pp. 427-437, 2009.

\section{AUTHORS PROFILE}

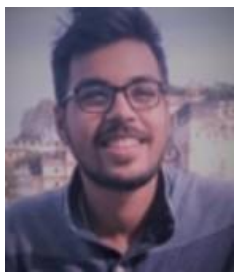

Prakhar Jain pursued his bachelor's degree in Computer Science and Engineering from Maulana Azad National Institute of Technology, Bhopal, India. His research interests include Machine Learning, Computer Vision, Internet of Things (IoT) and Pattern Recognition.

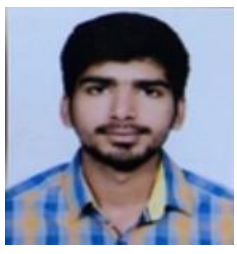

Yeshveer Singh Yadav is a M-Tech Student at Maulana Azad National Institute of Technology. He did B-Tech in Computer Science and Engineering in 2017 from A.P.J Abdul Kalam Technical University, Uttar Pradesh. His research interests include Internet of things (IoT) and Wireless Sensor Network

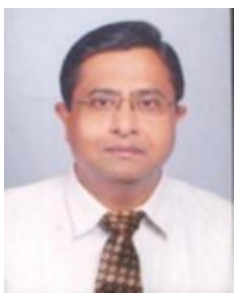

Nilay Khare is a professor at Maulana Azad National Institute of Technology. He did MTech. in Computer Science and Engineering from IIT Delhi. He received Ph.D. degree in Computer Science and Engineering. He has been professor in Department of Technical Education, Government of Madhya Pradesh, India.

He has also worked as head of State Project Facilitation Unit, Government of Madhya Pradesh, India and as head of department of Computer Science and Engineering, Rajiv Gandhi Technological University, Bhopal, Madhya Pradesh, India. He has published more than 50 papers in national and international journals. He is member of Indian Society of Technical Education (ISTE) and Computer Society of India (CSI). His research areas include Wireless Networks and Theoretical Computer Science.
Published By: Blue Eyes Intelligence Engineering \& Sciences Publication (C) Copyright: All rights reserved.

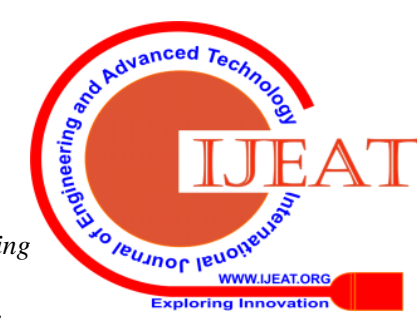

